\title{
Efficacy of simvastatin on carotid atherosclerotic plaque and its effects on serum inflammatory factors and cardiocerebrovascular events in elderly patients
}

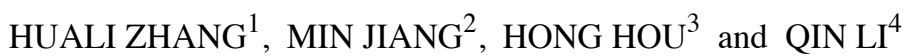 \\ ${ }^{1}$ Department of Geriatrics, Gansu Institute of Traditional Chinese Medicine, Gansu Provincial Hospital of \\ Traditional Chinese Medicine, Lanzhou, Gansu 730050; ${ }^{2}$ Department of Nephropathy, People's Hospital of Wuwei, \\ Wuwei, Gansu 733000; ${ }^{3}$ ECG Room, Xi'an no. 3 Hospital, Xi'an, Shaanxi 710008; ${ }^{4}$ Department of Geriatrics, \\ Baoji City People's Hospital, Baoji, Shaanxi 721000, P.R. China
}

Received September 17, 2019; Accepted March 18, 2021

DOI: $10.3892 / \mathrm{etm} .2021 .10251$

\begin{abstract}
To investigate the efficacy of simvastatin on carotid atherosclerotic plaque (CAP) and its effects on serum inflammatory factors and cardiocerebrovascular events in elderly patients, 130 elderly patients with CAP were randomly divided into observation $(n=65)$ and control groups $(n=65)$. The control group was treated with $75 \mathrm{mg} /$ day aspirin enteric-coated tablets, and the observation group was administered additional $20 \mathrm{mg} /$ day simvastatin. Serum total cholesterol, triglyceride, and high- and low-density lipoprotein cholesterol levels (evaluated via the endpoint method) were determined in both groups. Furthermore, the length, thickness and number of CAPs was measured using color Doppler ultrasonography. In addition, levels of inflammatory biomarkers including high-sensitivity C-reactive protein (hs-CRP), tumor necrosis factor- $\alpha$ (TNF- $\alpha$ ), interleukin-6 (IL-6), nitric oxide, D-dimer and fibrinogen, as well as change in microemboli count, were also compared After treatment, the observation group exhibited a significant reduction in size, thickness, and number of CAP and intima-media thickness compared with before treatment. However, no significant difference was found in the indicators of CAPs in the control group before and after treatment. The levels of total cholesterol, triglyceride, and low-density lipid cholesterol decreased, while high-density lipid cholesterol increased in the observation group after treatment, with notable changes in the observation group compared with in the control group. Overall response rate was higher in the observation group compared with the control group. TNF- $\alpha$, IL-6, and hs-CRP levels in the observation group decreased after
\end{abstract}

Correspondence to: Mrs. Qin Li, Department of Geriatrics, Baoji City People's Hospital, 24 Xinhua Lane, Jinger Road, Weibin, Baoji, Shaanxi 721000, P.R. China

E-mail: liiiqin@163.com

Key words: simvastatin, carotid atherosclerotic plaque, serum inflammatory factor, cardiocerebrovascular event, microemboli treatment compared with those before treatment and those in the control group. Furthermore, the rate of microemboli positivity was lower in the observation group than in the control group. Moreover, the overall incidence of acute cardiocerebrovascular events was lower in the observation group than in the control group. Therefore, it was demonstrated that simvastatin can reduce blood lipid levels, decrease the quantity and size of plaques, alleviate inflammatory response, reduce microemboli formation and reduce the risk of cardiocerebrovascular events in elderly patients with CAP.

\section{Introduction}

Atherosclerosis primarily refers to the deposition of fatty streaks and lipid spots in large elastic arteries, including medium-sized elastic arteries (cerebral and coronary arteries) and aortas in the blood circulatory system, which results in the development of fibrous atherosclerotic plaque and atheromatous lesions. Some of these changes may deteriorate the elasticity of the arterial wall, block the lumen and induce cardiocerebrovascular diseases, such as coronary heart disease and cerebral infarction (CI) (1). Carotid atherosclerotic plaque (CAP) is an independent risk factor for cardiocerebrovascular events (2). Chen et al (3) observed atherosclerosis in patients with CI using high-resolution MRI, and the results showed that the proportion of severe stenosis of offending vessels and plaque load in patients with recurrent CI were higher than those in patients with primary CI; logistic regression analysis also showed that severe lumen stenosis was a risk factor for the recurrence of CI. Tang and Luo (4) pointed out that the incidence of moderate carotid artery stenosis, plaque detection rate, and incidence of soft plaques in patients with myocardial infarction and hypertension were higher than those in the control group without myocardial infarction and hypertension, and the degree of carotid atherosclerotic vascular stenosis and plaque stability can reflect the risk of cardiovascular disease in patients to a certain extent, thus the degree of carotid atherosclerosis can be used as a predictor of cardiovascular diseases, including myocardial infarction. Therefore, early diagnosis and treatment of atherosclerotic plaques via promotive and 
effective measures are clinically significant in preventing the development of severe cardiocerebrovascular diseases.

Previous studies have revealed that regular oral administration of lipid-lowering agents, such as statins, effectively reduces blood lipid levels, and consequently, the incidence of cardiocerebrovascular events in patients with CAP (5). Simvastatin is commonly used in clinical practice to lower blood lipid levels, improve vascular endothelial function and stabilize atherosclerotic plaques in patients with hyperlipidemia (6). Zhang et al (7) found that the effective rate of simvastatin in the treatment of patients with atherosclerosis was $89.80 \%$, which was equivalent to that of atorvastatin calcium, but that the serum high-sensitivity C-reactive protein (hs-CPR), carotid intima-media thickness (IMT), cystatin C, triglyceride (TG), total cholesterol (TC) and low-density lipid cholesterol (LDL-C) in simvastatin group were significantly lower than those in the atorvastatin calcium group, suggesting that simvastatin has a significant effect on patients with atherosclerosis. Compared with other statins, simvastatin is primarily characterized by mild adverse reactions and better tolerance in patients. To further validate the therapeutic effect of simvastatin on CAP, the present study was designed to investigate the efficacy of simvastatin in the treatment of elderly patients with carotid atherosclerosis, and to further observe the effect of simvastatin on serum inflammatory factors and cardiocerebrovascular events.

\section{Materials and methods}

General characteristics. A total of 130 elderly patients diagnosed with CAP in the outpatient department of Baoji City People's Hospital (Baoji, China) were included. The inclusion criteria were as follows: i) Patients with soft CAP as evidenced by carotid artery color Doppler ultrasound, with $<70 \%$ arterial luminal stenosis; ii) those who met the diagnostic standards for CAP revised by the Fourth National Academic Conference for Cerebrovascular Diseases (8); iii) those who were willing to follow the doctor's advice and adhere to long-term medication without drop-out cases midway; and iv) those who signed informed consent and volunteered to participate in the present study. Patients with the following conditions were excluded: i) Previous treatment using statins or other lipid-lowering agents 1 month before enrollment in the study; ii) severe carotid stenosis with a carotid stenosis rate of $>70 \%$; iii) statin allergy, uncontrolled hypertension, familial hypercholesterolemia or secondary hypercholesterolemia; iv) infectious diseases, severe gastrointestinal (GI) diseases, or history of GI surgery that may affect drug absorption; v) severe liver and kidney dysfunction or heart failure; and vi) malignant tumors or bleeding within plaques. The present study was approved by the Medical Ethics Committee of Baoji City People's Hospital (Baoji, China). Patients were randomly divided into control and observation groups ( $\mathrm{n}=65$ per group).

Treatment plan. Functional indicators of the heart, liver, and kidney were measured for each patient before enrollment. If necessary, antihypertensive and hypoglycemic agents were administered to patients, and dietary therapy was administered to control blood lipid levels. In the present study, the control group was administered $75 \mathrm{mg}$ oral aspirin enteric-coated tablets daily (Harbin Tiandi Pharmaceutical Co., Ltd.;
National Medicine Permission no. H23023503; drug specification, $0.25 \mathrm{~g}$ ) and, based on the treatment administered to the control group, the observation group was administered additional $20 \mathrm{mg}$ oral simvastatin tablets every night (Shanghai Xinyi Wanxiang Pharmaceutical Co., Ltd.; National Medicine Permission no. H19980174; drug specification, 5 mg). Both groups were treated continuously for 12 months.

\section{Outcome measurements}

Determination of blood lipid. In total, $5 \mathrm{ml}$ fasting venous blood in the early morning was collected from all patients before and 12 months after treatment. TC and TG levels were determined using the oxidase method; high-density lipid cholesterol (HDL-C) and LDL-C levels were determined using the endpoint method.

Detection of carotid ultrasound indexes. The shape and internal echo of the bilateral common carotid, internal carotid, external carotid and vertebral arteries, as well as the length, thickness (IMT) and number of arterial plaques were measured and compared before and 12 months after treatment using HPSONOS-5500 color ultrasound Doppler instrument (Hewlett-Packard) with high-frequency L-7540 probes at a frequency of $4-100 \mathrm{mHz}$. The transverse and longitudinal ultrasound imaging of the common carotid artery and the posterior wall of the common carotid artery is shown as two parallel lines separated by lower echo, and the distance between the two lines was recorded as IMT. The image $1 \mathrm{~cm}$ below the carotid sinus was frozen and measured 5 times in total, and the average value of the left and right side of 10 measurements was recorded as the final IMT.

The criteria for efficacy evaluation were as follows (9). Significant response, $\geq 90 \%$ plaque reduction or shrinkage or disappearance; response, $30-89 \%$ plaque reduction or shrinkage; non-response, $<30 \%$ plaque reduction or shrinkage or occurrence of cardiocerebrovascular events. Overall response rate $(\mathrm{ORR})=($ significant response + response $) / 65 \times 100 \%$.

Measurement of inflammatory factors. Additionally, $3 \mathrm{ml}$ fasting venous blood before and 12 months after treatment was collected from all patients, and serum was separated by centrifugation at $1,000 \mathrm{x}$ for $5 \mathrm{~min}$ at $4^{\circ} \mathrm{C}$. hs-CRP, tumor necrosis factor- $\alpha$ (TNF- $\alpha$ ) and interleukin-6 (IL-6) levels were determined using enzyme-linked immunosorbent assay (ELISA) kits (R\&D Systems, Inc.) of hs-CRP (cat. no. 2119), TNF- $\alpha$ (cat. no. 1576), and IL-6 (cat. no. 3166). All operations strictly followed the manufacturer's instructions.

Detection of nitric oxide (NO), D-dimer and fibrinogen. $5 \mathrm{ml}$ venous blood before and 12 months after treatment was collected from all patients. NO level was determined using an ELISA kit (cat. no. 7945; Roche Diagnostics), and plasma D-dimer and fibrinogen levels were determined using thrombin turbidimetry with a Sysmex CA-7000 automatic coagulation analyzer (Sysmex Shanghai Ltd.) with default parameters. All operations strictly followed the manufacturer's instructions.

Detection of microemboli. The inspection instrument used was Multi-Dop X4 TCD instrument (DWL) with 2-MHz pulse wave-measuring probes. Patients were informed to maintain 
Table I. Comparison of general data between the observation $(n=65)$ and control $(n=65)$ groups.

\begin{tabular}{|c|c|c|c|c|c|c|c|c|c|c|c|c|}
\hline \multirow[b]{3}{*}{ Group } & \multirow{2}{*}{\multicolumn{2}{|c|}{ Sex }} & \multirow{3}{*}{$\begin{array}{c}\text { Mean age } \\
\text { (years) }\end{array}$} & \multirow{2}{*}{\multicolumn{4}{|c|}{ Primary disease }} & \multicolumn{5}{|c|}{ Original medication } \\
\hline & & & & & & & & & & & & \\
\hline & M & $\mathrm{F}$ & & CHD & Hypertension & Diabetes & CI & Aspirin & antagonist & ACEI & $\beta$-Blocker & ester \\
\hline Observation & 35 & 30 & $74.71 \pm 5.12$ & 17 & 22 & 15 & 20 & 16 & 15 & 21 & 20 & 28 \\
\hline Control & 37 & 28 & $73.88 \pm 5.86$ & 20 & 24 & 13 & 18 & 17 & 13 & 23 & 17 & 25 \\
\hline
\end{tabular}

Date are expressed as mean \pm SD or $\mathrm{n}$. ACEI, angiotensin-converting enzyme inhibitor; CHD, coronary heart disease; CI, cerebral infarction.

the supine position, and the probes were placed on both sides of their head to obtain the optimal blood flow signal of the middle cerebral artery. The parameters were set to $45-38 \mathrm{~mm}$ in depth, $5 \mathrm{~mm}$ in spacing and $8 \mathrm{ml}$ in volume. After monitoring for $30 \mathrm{~min}$, the spectrum and audio frequency of microemboli were analyzed using transcranial Doppler emboli detection software (version 8.00) (DWL). The diagnostic criteria for positive microemboli included the following: i) duration $<300 \mathrm{~ms}$; ii) $\geq 6 \mathrm{~dB}$ of signal intensity higher than background Doppler blood flow spectrum; iii) single-phase microembolic signal and above the baseline; iv) a 'tweet'-like audio signal; and v) random appearance during the cardiac cycle.

Record of cardiocerebrovascular events. The incidence of cardiocerebrovascular events during treatment was recorded. The data were collected during the treatment period, which was the incidence during 12 months of continuous treatment.

Statistical analysis. Data were analyzed using SPSS 23.0 (IBM Corp.). The mean between the two groups was compared using two independent samples t-test, and paired t-test was used to compare the mean before and after intervention within the same group. A mixed two-way ANOVA followed by Bonferroni's correction was used for within-group and between-group comparisons. Numerical data are presented as rates or percentages and analyzed using the $\chi^{2}$ test. $\mathrm{P}<0.05$ was considered to indicate a statistically significant difference.

\section{Results}

Comparison of baseline data between the two groups. No significant differences were found in terms of sex, age, primary diseases and original medication between the two groups, indicating comparability between them $(\mathrm{P}>0.05$; Table I).

Simvastatin improves CAP in elderly patients. Before treatment, there was no statistically significant difference in the size, thickness, and number of CAP and IMT between the two groups $(\mathrm{P}>0.05)$. In the observation group, intragroup comparison showed that the size $(\mathrm{P}<0.01)$, thickness $(\mathrm{P}<0.001)$, and number of CAP $(\mathrm{P}<0.05)$ and IMT $(\mathrm{P}<0.001)$ significantly decreased after treatment, compared with before treatment. However, no significant differences were noted in these indices in the intragroup comparison of the control group before and after treatment, suggesting that simvastatin improved CAP in elderly patients in terms of the size and thickness of CAP and IMT (Fig. 1).
Simvastatin improves blood lipid levels in elderly patients with CAP. Intragroup comparison of the observation group revealed that TC, TG and LDL-C levels significantly decreased, and HDL-C level significantly increased after treatment compared with before treatment $(\mathrm{P}<0.001)$. However, no significant differences were found in these indices in the intragroup comparison of the control group before and after treatment. These results suggested that simvastatin improved blood lipid levels in elderly patients with CAP (Fig. 2).

Clinical efficacy. ORR of the observation group was 93.85\%, which was significantly higher than that of the control group $(80.00 \%$; $\mathrm{P}<0.05)$, suggesting that simvastatin improved the characteristics of the indices of arterial plaques in elderly patients with CAP (Table II).

Simvastatin reduces serum inflammatory factor levels in elderly patients with CAP. Intergroup comparison revealed no significant differences in TNF- $\alpha$, IL- 6 and hs-CRP levels before treatment $(\mathrm{P}>0.05)$. Intragroup comparison of both groups indicated that TNF- $\alpha$, IL- 6 and hs-CRP levels significantly decreased after treatment compared with before treatment $(\mathrm{P}<0.001)$. Intergroup comparison after treatment revealed that TNF- $\alpha$, IL- 6 and hs-CRP levels were significantly lower in the observation group than in the control group $(\mathrm{P}<0.001)$. These results indicate that simvastatin reduces inflammatory factor levels in elderly patients with CAP (Fig. 3).

Simvastatin increases NO levels and decreases D-dimer and fibrinogen levels in elderly patients with CAP. Before treatment, there was no statistically significant difference in NO, D-dimer, and fibrinogen levels between the two groups $(\mathrm{P}>0.05)$. Intragroup comparison of the observation group revealed that NO level significantly increased $(\mathrm{P}<0.001)$, and D-dimer $(\mathrm{P}<0.001)$ and fibrinogen $(\mathrm{P}<0.05)$ levels significantly decreased after treatment compared with before treatment $(\mathrm{P}<0.05)$. However, no significant differences were noted in terms of NO, D-dimer and fibrinogen levels in the control group before and after treatment $(\mathrm{P}>0.05)$, suggesting that simvastatin improves NO, D-dimer and fibrinogen levels in elderly patients with CAP (Fig. 4).

Simvastatin reduces microemboli formation in elderly patients with CAP. The rate of microemboli positivity in the observation group after treatment was significantly lower than that before treatment and in the control group $(\mathrm{P}<0.01)$, 


$$
\text { A }
$$
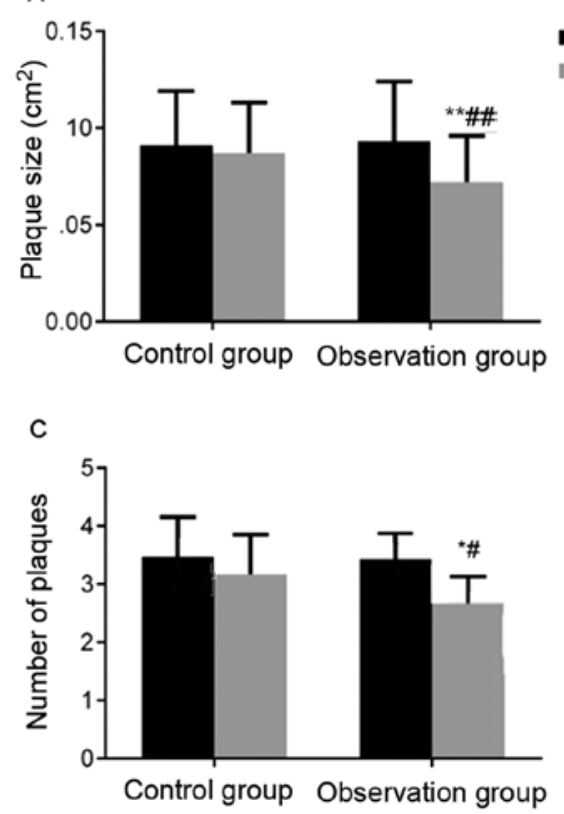
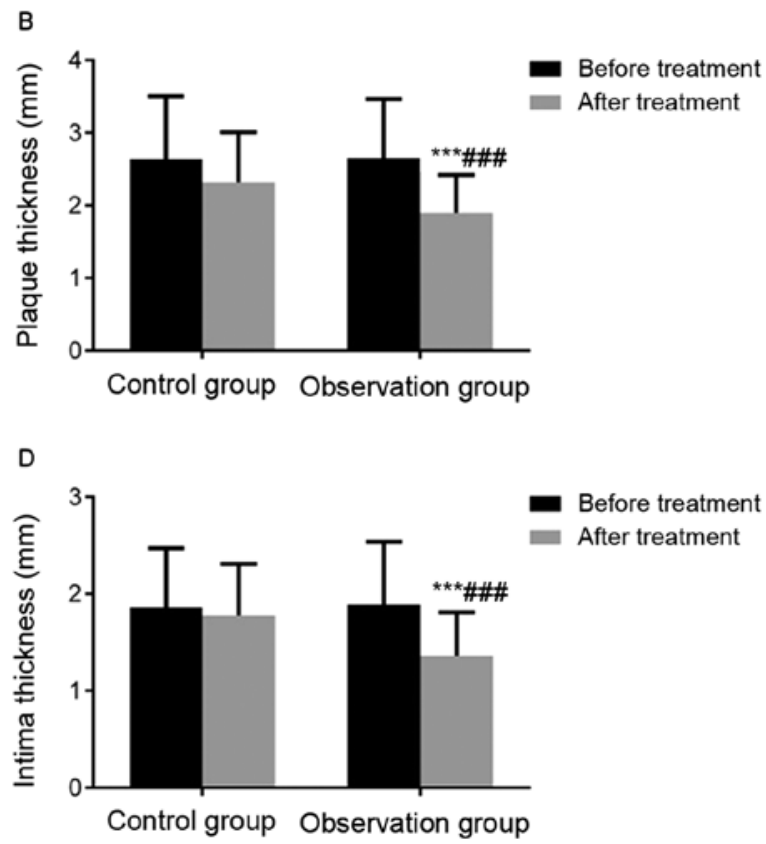

Figure 1. Comparison of color Doppler ultrasound indicators of the carotid artery before and after treatment in the two groups. (A) Plaque size; (B) plaque thickness; (C) number of plaques; and (D) intima thickness. $\mathrm{P}<0.05,{ }^{* *} \mathrm{P}<0.01,{ }^{* * *} \mathrm{P}<0.001$ vs. before treatment; ${ }^{\#} \mathrm{P}<0.05,{ }^{\# \#} \mathrm{P}<0.01,{ }^{\# \# \#} \mathrm{P}<0.001$ vs. control group after treatment.

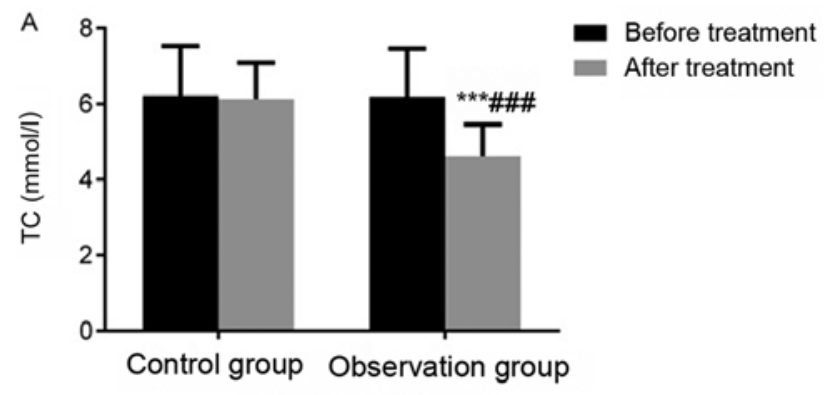

C

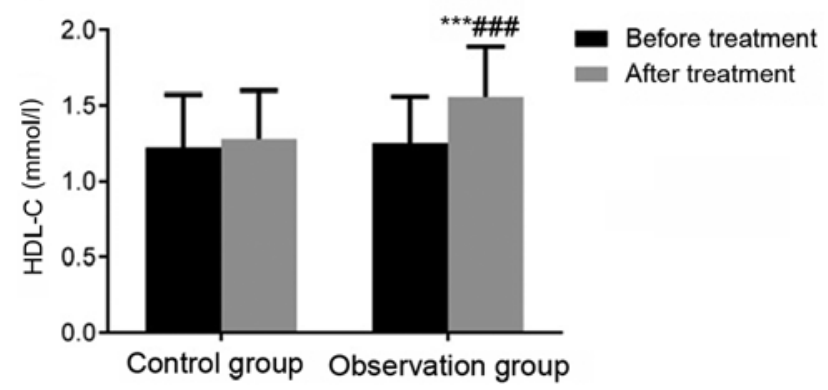

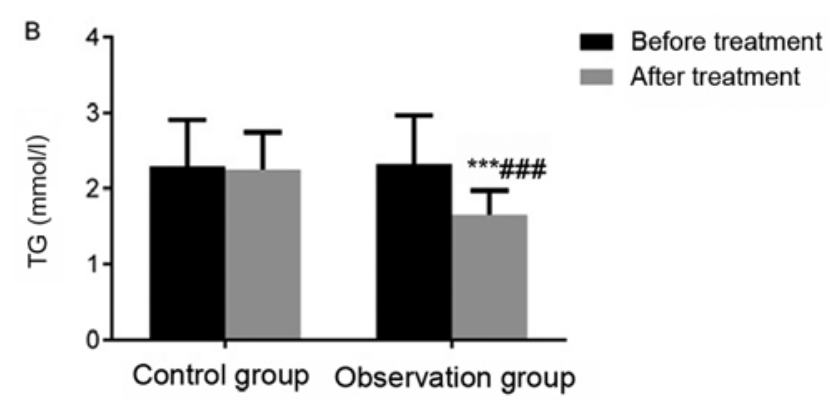

D

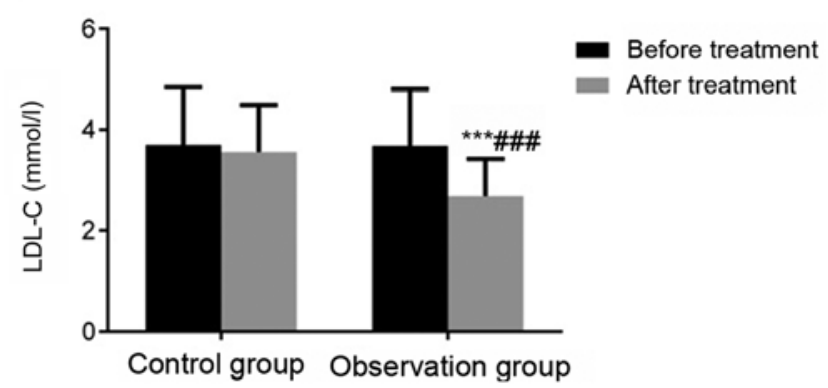

Figure 2. Comparison of blood lipid levels before and after treatment in the two groups. ${ }^{* * *} \mathrm{P}<0.001$ vs. before treatment; ${ }^{\# \#} \mathrm{P}<0.001$ vs. control group after treatment. TC, total cholesterol; TG, triglyceride; HDL-C, high-density lipid cholesterol; LDL-C, low-density lipid cholesterol. (A) TC; (B) TG; (C) HDL-C; (D) LDL-C.

suggesting that simvastatin reduced microemboli formation in elderly patients with CAP (Table III).

Simvastatin reduces the incidence of acute cardiocerebrovascular events in elderly patients with CAP. The incidence of acute cardiocerebrovascular events was significantly lower in the observation group than in the control group $(\mathrm{P}<0.01)$, suggesting that simvastatin reduces the incidence of acute cardiocerebrovascular events in elderly patients with CAP (Table IV).

\section{Discussion}

Atherosclerosis is a chronic systemic disease commonly found in the medium-aged and elderly population, and it is reported that the detection rate of atherosclerosis in individuals aged 40-49 years is $58.36 \%$, and its incidence increases with age (10). Carotid arteries, including bilateral common carotid arteries, bifurcation of the common carotid artery and extracranial segment of the internal carotid artery, are the common sites 
Table II. Comparison of clinical efficacy between the observation $(n=65)$ and control $(n=65)$ groups.

\begin{tabular}{lcccc}
\hline Group & Significant response & Response & Non-response & Overall response rate \\
\hline Control group & $30(46.15)$ & $22(33.85)$ & $13(20.00)$ & $52(80.00)$ \\
Observation group & $36(55.38)$ & $25(38.46)$ & $4(6.15)$ & $61(93.85)^{\mathrm{a}}$ \\
\hline
\end{tabular}

Data are expressed as $\mathrm{n}(\%)$. ${ }^{\mathrm{a}}<0.05$ vs. the control group after treatment.

A

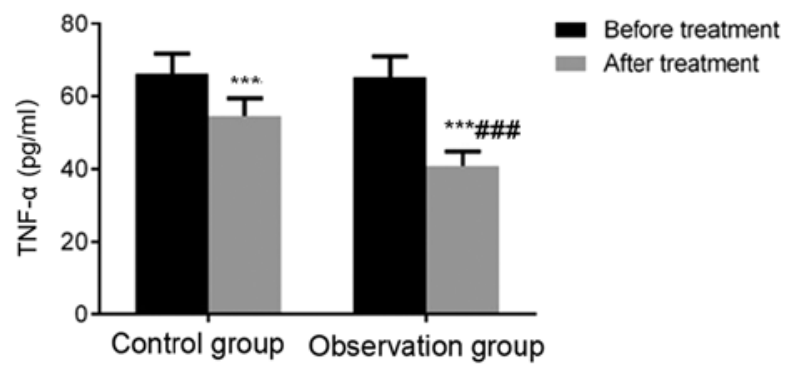

C

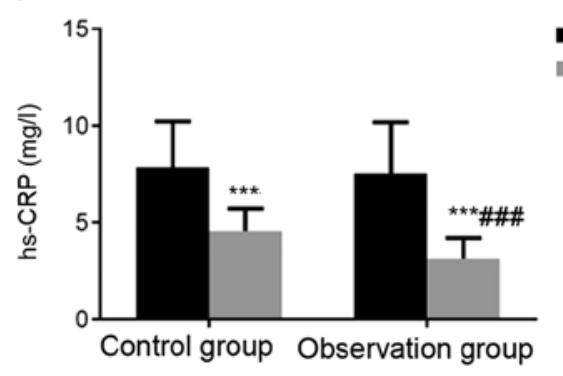

B

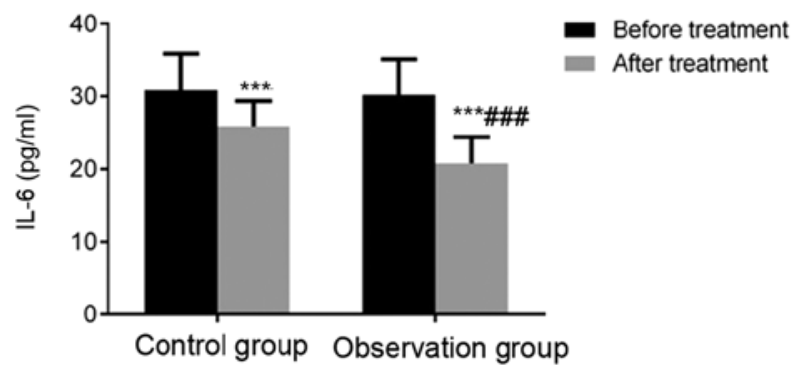

Figure 3. Comparison of serum inflammatory factors between the two groups (mean $\pm \mathrm{SD}$ ). ${ }^{* * * *} \mathrm{P}<0.001 \mathrm{vs}$. before treatment; ${ }^{\# \# \#} \mathrm{P}<0.001 \mathrm{vs}$. control group after treatment. TNF- $\alpha$, tumor necrosis factor- $\alpha$; IL-6, interleukin-6; hs-CRP, high-sensitivity C-reactive protein. (A) TNF- $\alpha$; (B) IL-6; (C) hs-CRP.

A

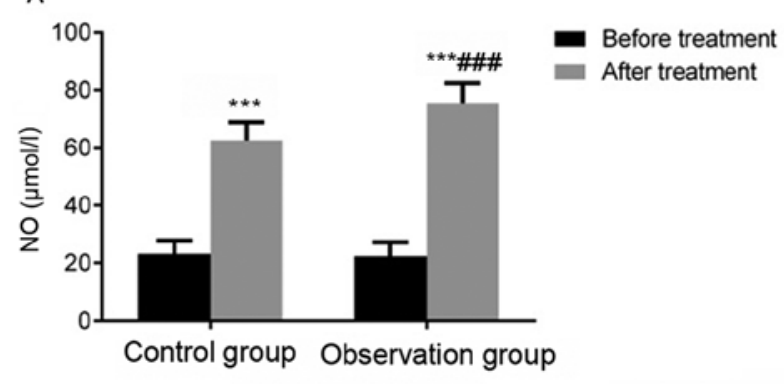

C

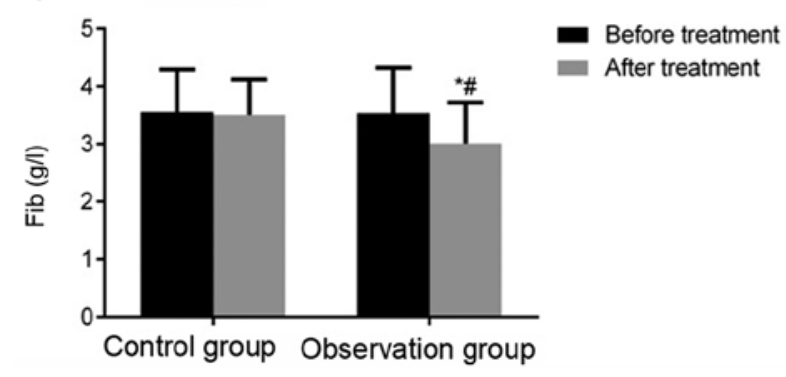

B

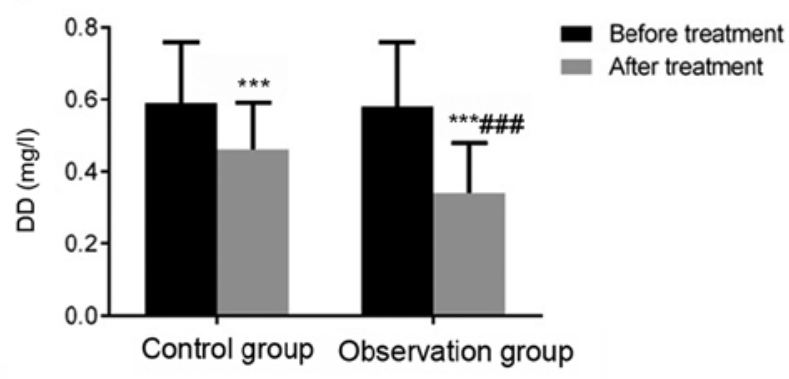

Figure 4. Comparison of serum nitric oxide, D-dimer, and fibrinogen levels between the two groups. (A) NO; (B) DD; (C) Fib. (mean \pm SD). "P<0.05, ${ }^{* * * *} \mathrm{P}<0.001$ vs. before treatment; ${ }^{~} \mathrm{P}<0.05,{ }^{\# \# \#} \mathrm{P}<0.001$ vs. control group after treatment. NO, nitric oxide; Fib, fibrinogen; DD, D-dimer. 
Table III. Comparison of microemboli count between the observation $(n=65)$ and control $(n=65)$ groups.

\begin{tabular}{lrrrrr}
\hline & \multicolumn{2}{c}{$\begin{array}{c}\text { Before } \\
\text { treatment }\end{array}$} & & \multicolumn{2}{c}{$\begin{array}{c}\text { After } \\
\text { treatment }\end{array}$} \\
\cline { 2 - 3 } \cline { 6 - 7 } Groups & $\mathrm{n}$ & $\%$ & & $\mathrm{n}$ & $\%$ \\
\hline Control group & 27 & 41.54 & & 20 & 30.77 \\
Observation group & 25 & 38.46 & & 6 & $9.23^{\mathrm{a}, \mathrm{b}}$ \\
\hline
\end{tabular}

Data are expressed as mean $\pm \mathrm{SD}, \%$. ${ }^{\mathrm{a}} \mathrm{P}<0.001 \mathrm{vs}$. before treatment; ${ }^{\mathrm{b}} \mathrm{P}<0.01$ vs. the control group after treatment.

Table IV. Comparison of acute cardiocerebrovascular events between the observation $(n=65)$ and control $(n=65)$ groups.

\begin{tabular}{|c|c|c|c|}
\hline Outcomes & $\begin{array}{l}\text { Observation } \\
\text { group }\end{array}$ & $\begin{array}{l}\text { Control } \\
\text { group }\end{array}$ & P-value \\
\hline All-cause death & (0) & $2(3.08)$ & $>0.01$ \\
\hline Myocardial infarction & $1(1.54)$ & $6(9.23)$ & $<0.01$ \\
\hline Stroke & $1(1.54)$ & $6(9.23)$ & $<0.01$ \\
\hline $\begin{array}{l}\text { Overall incidence of acute } \\
\text { cardiocerebrovascular events }\end{array}$ & $2(3.08)$ & $14(21.54)$ & $<0.01$ \\
\hline
\end{tabular}

Data are expressed as n (\%).

involved in atherosclerosis; furthermore IMT of $\geq 1.3 \mathrm{~mm}$ at any site is defined as a plaque, which can result in carotid stenosis and affects peripheral blood supply, causing adverse cardiocerebrovascular events (11). Factors responsible for CAP formation are complicated, and CAP is generally believed to be attributed to varying pathogeneses. The recognized mechanisms include mechanical (such as hypertension and carotid pulse pressure), inflammatory and physicochemical) factors (including lipid metabolism disorders and elevated fibrinogen) (12). An elevated cholesterol level caused by impaired lipid metabolism is considered the initiating factor for atherosclerosis, and chronic inflammation is critical for its continuous development (13). Therefore, regulating lipids, reducing the inflammatory response and decreasing fibrinogen levels may be key in improving disease-related symptoms and prognostic outcomes during the treatment of elderly patients with CAP.

Simvastatin is a member of the statin family. Numerous animal experiments and clinical trials have reported that statins have potent anti-atherosclerotic effects, and the underlying mechanisms may involve lipid-regulation and lipid-lowering effects, antiplatelet aggregation and thrombosis, maintenance of plaques, and improvement of endothelial function $(5,14,15)$. During lipid lowering, simvastatin effectively reduces the TC concentration in the blood and prevent lipid production, including TC, TG and LDL production, by lowering the reductase level of hydroxymethylglutaryl coenzyme A and blocking cholesterol synthesis (16). Relevant basic experiments and clinical studies have confirmed that simvastatin effectively reduces and eliminates CAPs, and consequently decreases the incidence of acute cardiocerebrovascular events to improve the prognosis of patients (17). In addition, simvastatin exhibits an anti-inflammatory effect primarilu via mechanisms such as reduction of inflammatory infiltrating cell count, inhibition of adhesion molecule function on isopentenylated $G$ protein and integrin, and reduction of CRP level (18). Currently, simvastatin is widely used in the clinical treatment of diseases such as cardiocerebrovascular diseases, atherosclerosis and myocardial ischemia (19). In the present study, the clinical control design was used to compare the effects of conventional therapy vs. simvastatin on CAP in elderly patients. The results indicated that the size, thickness and number of CAP and IMT were significantly decreased, TC, TG and LDL-C levels were significantly decreased, and HDL-C level was significantly increased in the observation group after treatment, compared with those in the control group. Furthermore, simvastatin treatment can reduce the size and number of CAPs with its lipid-lowering effect, thus producing a definite effect on CAP.

Chronic inflammatory response is one of the major mechanisms involved in carotid atherosclerosis in the elderly. After vascular endothelial injury, inflammatory response occurs in the vascular wall resulting in the production of various adhesion molecules and increased inflammatory factors such as TNF- $\alpha$, IL-6 and CRP. TNF- $\alpha$ and IL-6, among other inflammatory factors, enhance the local inflammatory response, resulting in plaque complications and decreased stability (20). Unstable plaques are more prone to breakage, shedding and metastasis, causing vascular embolization at the distal end and increasing the risk of cardiocerebrovascular events (21). CRP binds lipoproteins to activate the complement system and produce aggressive complexes by binding to ligands (apoptotic and necrotic cells, or phosphocholine of invading pathogens) and terminal protein $\mathrm{C}$, which can induce vascular damage (22). In addition, CRP may enhance the inflammatory response and aggravate vascular inflammation damage. Detection of hs-CRP is more sensitive than that of CRP, allowing the determination of even low concentrations of CRP (0.1-10 mg/l) (22). Prior evidence (23) supports that patients with diabetes with hs-CRP level of $\geq 2 \mathrm{mg} / \mathrm{l}$ are more likely to develop carotid atherosclerosis, and hs-CRP level was associated with the progression of carotid atherosclerosis and plaque count. In the current study, inflammatory factors (TNF- $\alpha$, IL-6 and hs-CRP) levels decreased in the observation group after treatment, suggesting that simvastatin reduced serum inflammatory factor levels and consequently the inflammatory response in elderly patients with CAP.

$\mathrm{NO}$ is a commonly used indicator for vascular endothelial function and is a vasodilator. Decreased NO level is associated with the degree of vascular endothelial cell injury (24). D-Dimer is one of the fibrin degradation products, which may be elevated due to coagulation dysfunction and microthrombosis (25). In addition, fibrinogen is one of the independent predictors of atherosclerosis and is primarily produced in the liver. Fibrinogen promotes platelet aggregation and thrombosis, which serves an important role in atherosclerotic lesion formation, and is associated with disease severity (26). The present study demonstrated that increased NO level and decreased D-dimer and fibrinogen levels in the observation group were more significant than those in the control group. Further, the decreased rate of microemboli positivity in the 
observation group was more notable than that in the control group after treatment. The aforementioned findings support that simvastatin improves serum NO level, protects vascular endothelium and reduces serum D-dimer and fibrinogen levels, which consequently improves blood coagulation status and eliminates microemboli.

Carotid atherosclerosis is the most critical risk factor for cardiocerebrovascular events and is an important index reflecting systemic arteriosclerosis due to the superficial location of the carotid artery and easy identification of CAP (27). A prospective cohort study showed that the incidences of all-cause death and cardiocerebrovascular events in patients with CAP were 5.50 and $3.80 \%$, respectively, which were higher than those in the non-plaque group (1.50 and $1.40 \%$, respectively) (28). A Cox proportional hazard regression model determined that CAP was an independent risk factor for all-cause death and cardiocerebrovascular events with 95\% confidence intervals of (1.160-2.395) and (1.312-2.876), respectively (28). The present study showed that the incidences of acute cardiocerebrovascular events were lower in the observation group than in the control group following treatment, indicating that simvastatin can prevent the development of acute cardiocerebrovascular events. This is possibly attributed to the multiple mechanisms of simvastatin in regulating lipids, reducing inflammatory response, eliminating microemboli, protecting vascular endothelium and improving blood coagulation status, thus facilitating the reduction of mortality and prevention of acute heart and brain events.

In summary, continuous use of statins may stabilize and dissolve CAPs in elderly patients and reduce acute cardiocerebrovascular events by lowering blood lipid levels. It was demonstrated that simvastatin reduces blood lipid levels, number and size of plaques, the inflammatory response, microemboli formation in elderly patients with CAP and the risk of cardiovascular events, which is the primary novelty of this study. The present findings suggest that simvastatin treatment for elderly patients with atherosclerotic plaque can effectively reduce the risk of cardiocerebrovascular events. The present study was a preliminary efficacy study, and only the changes in the levels of inflammatory factors and other indicators between the two groups were preliminarily observed. Whether there is correlation between factors, and whether simvastatin primarily functions through that signaling pathway was not included in the present study, and the specific signaling pathways mediated by these factors are still unknown. In addition, the observation time was set to 1 year, and the research time is relatively short. In future studies, the research time should be increased, and the main pathway of its function should be detected. Part of the study on the mechanism is conjecture, and the correctness of the aforementioned hypotheses should be verified in a multi-center study with and expanded sample size and a prolonged observation time.

\section{Acknowledgements}

Not applicable.

\section{Funding}

No funding was received.

\section{Availability of data and materials}

The datasets used and/or analyzed during the current study are available from the corresponding author on reasonable request.

\section{Authors' contributions}

HZ and QL conceived the study and designed the experiments. $\mathrm{MJ}$ and $\mathrm{HH}$ contributed to data collection, performed data analysis and interpreted the results. $\mathrm{HZ}$ and QL confirmed the authenticity of all the raw data. HZ wrote the manuscript. QL contributed to the critical revision of article. All authors read and approved the final manuscript.

\section{Ethics approval and consent to participate}

The present study was approved by the Medical Ethics Committee of Baoji City People's Hospital (Baoji, China) and all patients signed informed consent and volunteered to participate in this study.

\section{Patient consent for publication}

Not applicable.

\section{Competing interests}

The authors declare that they have no competing interests.

\section{References}

1. Rafailidis V, Chryssogonidis I, Tegos T, Kouskouras K and Charitanti-Kouridou A: Imaging of the ulcerated carotid atherosclerotic plaque: A review of the literature. Insights Imaging 8: 213-225, 2017.

2. Kwee R, Oostenbrugge R, Hofstra L, van Engelshoven J, Mess W Wildberger J and Kooi ME: Noninvasive Imaging of Carotid Atherosclerosis, pp497-525, 2011.

3. Chen SX, Hong GX, Li ZH and Yang ZY: High resolution MRI observation on intracranial atherosclerotic plaque and risk factors in patients with recurrent cerebral infarction. Chin J Med Imaging Technol 36: 2020.

4. Tang QH and Luo KQ: Color doppler ultrasound in detection of carotid atherosclerotic stenosis degree and plaque distribution in patients with myocardial infarction associated by hypertension. Med Pharm J Chin PLA 31: 44-47, 2019.

5. Eilenberg W, Stojkovic S, Kaider A, Kozakowski N, Domenig CM, Burghuber C, Nanobachvili J, Huber K, Klinger M, Neumayer C, et al: NGAL and MMP-9/NGAL as biomarkers of plaque vulnerability and targets of statins in patients with carotid atherosclerosis. Clin Chem Lab Med 56: 147-156, 2017.

6. Huang HY and Yan WM: Comparison of the efficacy and safety of 4 kinds of statins in the treatment of atherosclerosis. China Pharm 27: 5100-5103, 2016.

7. Zhang N, Ma Y and Wang R: Pharmacoeconomic evaluation of simvastatin capsules and atorvastatin calcium tablets in the treatment of patients with atherosclerosis. China J Pharm Econ 16: 31-34, 2021.

8. Wang WW and Wang XD: Summary of the 6th national cerebrovascular disease academic conference. Chin J Neurol 37: 346-348, 2004.

9. Zhao X, Hippe DS, Li R, Canton GM, Sui B, Song Y, Li F, Xue Y, Sun J, Yamada K, et al: Prevalence and characteristics of carotid artery high-risk atherosclerotic plaques in chinese patients with cerebrovascular symptoms: A Chinese atherosclerosis risk evaluation ii study. J Am Heart Assoc 6: e005831, 2017. 
10. Cao Y, Sun Y, Zhou B, Zhao H, Zhu Y, Xu J and Liu X: Atherosclerotic plaque burden of middle cerebral artery and extracranial carotid artery characterized by MRI in patients with acute ischemic stroke in China: Association and clinical relevance. Neurol Res 39: 344-350, 2017.

11. Alloza I, Goikuria H, Idro JL, Triviño JC, Fernández Velasco JM, Elizagaray E, García-Barcina M, Montoya-Murillo G, Sarasola E, Vega Manrique R, et al: RNAseq based transcriptomics study of SMCs from carotid atherosclerotic plaque: BMP2 and IDs proteins are crucial regulators of plaque stability. Sci Rep 7: $3470,2017$.

12. Ni T, Chen M, Yang K, Shao J, Fu Y and Zhou W: Association of CD147 genetic polymorphisms with carotid atherosclerotic plaques in a Han Chinese population with cerebral infarction. Thromb Res 156: 29-35, 2017.

13. Schaefer CA, Blatzheim AK, Passon SG, Pausewang KS, Schahab N, Nickenig G, Skowasch D, Schueler R, Hammerstingl C and Pingel S: Modulation of carotid strain by statin therapy in atherosclerosis patients. Vasa 46: 108-115, 2017.

14. Natarajan P, Young R, Stitziel NO, Padmanabhan S, Baber U, Mehran R, Sartori S, Fuster V, Reilly DF, Butterworth A, et al: Polygenic risk score identifies subgroup with higher burden of atherosclerosis and greater relative benefit from statin therapy in the primary prevention setting. Circulation 135: 2091-2101, 2017

15. Kocaman SA, Baysan O, Cetin M, Kayhan Altuner T, Polat Ocaklı E, Durakoğlugil ME, Erdoğan T and Karaoğuz MR: An increase in epicardial adipose tissue is strongly associated with carotid-intima media thickness and atherosclerotic plaque, but LDL only with the plaque. Anatol J Cardiol 17: 56-63, 2017.

16. Ahmed AM: Inhibition of inducible nitric oxide synthase (iNOS) by simvastatin attenuates cardiac hypertrophy in rats. Folia Morphol (Warsz) 76: 15-27, 2017.

17. Ngo-Metzger Q and Gottfredson R: Statin Use for the primary prevention of cardiovascular disease in adults. Am Fam Physician 96: 805-806, 2017.

18. Feng Y, Lei B, Zhang F, Niu L, Zhang $\mathrm{H}$ and Zhang M: Anti-inflammatory effects of simvastatin during the resolution phase of experimentally formed venous thrombi. J Investig Med 65: 999-1007, 2017.

19. Środa-Pomianek K, Michalak K, Palko-Łabuz A, Uryga A, Szczęśniak-Sięga B and Wesołowska O: Simvastatin strongly augments proapoptotic, anti-inflammatory and cytotoxic activity of oxicam derivatives in doxorubicin-resistant colon cancer cells. Anticancer Res 39: 727-734, 2019.
20. Stefanutti C, Mazza F, Pasqualetti D, Di Giacomo S, Watts GF, Massari MS, de Neve J, Morozzi C and Fischer M: Lipoprotein apheresis downregulates IL- $1 \alpha$, IL- 6 and TNF- $\alpha$ mRNA expression in severe dyslipidaemia. Atheroscler Suppl 30: 200-208, 2017.

21. Poredos P, Spirkoska A, Lezaic L, Mijovski MB and Jezovnik MK: Patients with an inflamed atherosclerotic plaque have increased levels of circulating inflammatory Markers. J Atheroscler Thromb 24: 39-46, 2017.

22. Braig D, Nero TL, Koch HG, Kaiser B, Wang X, Thiele JR, Morton CJ, Zeller J, Kiefer J, Potempa LA, et al: Transitional changes in the CRP structure lead to the exposure of proinflammatory binding sites. Nat Commun 8: 14188, 2017.

23. Pleskovič A, Letonja M, Vujkovac AC, Nikolajević Starčević J, Gazdikova K, Caprnda M, Gaspar L, Kruzliak P and Petrovič D: $\mathrm{C}$-reactive protein as a marker of progression of carotid atherosclerosis in subjects with type 2 diabetes mellitus. Vasa 46: 187-192, 2017.

24. Jorgensen AL: Contrast-induced nephropathy: Pathophysiology and preventive strategies. Crit Care Nurse 33: 37-46, 2013.

25. Yang N,Hao J and Zhang D: Antithrombin III and D-dimer levels as indicators of disease severity in patients with hyperlipidaemic or biliary acute pancreatitis. J Int Med Res 45: 147-158, 2017.

26. Schreiner PJ, Appiah D and Folsom AR: Gamma prime $\left(\gamma^{\prime}\right)$ fibrinogen and carotid intima-media thickness: The atherosclerosis risk in communities study. Blood Coagul Fibrinolysis 28: 665-669, 2017.

27. Clarke R, Du H, Kurmi O, Parish S, Yang M, Arnold M, Guo Y, Bian Z, Wang L, Chen Y, et al: Burden of carotid artery atherosclerosis in Chinese adults: Implications for future risk of cardiovascular diseases. Eur J Prev Cardiol 24: 647-656, 2017.

28. Li W, Ma F, Jiang YM, Li JJ, Song L, Chen SH, Liu XM, Li XQ and Wu SL: Association between carotid artery plaques and all-cause mortality and cardiovascular events. Zhonghua Xin Xue Guan Bing Za Zhi 45: 1086-1090, 2017 (In Chinese).

This work is licensed under a Creative Commons Attribution-NonCommercial-NoDerivatives 4.0 International (CC BY-NC-ND 4.0) License. 